\title{
A Note on the Local Stability Theory for Caputo Fractional Planar System
}

\author{
Marvin Hoti
}

October 8, 2020

Ryerson University, 350 Victoria St, Toronto, ON M5B 2K3

Email: marvin.hoti@ryerson.ca

Subject class 2010: 34A08, 37B55, $03 \mathrm{C} 45$.

Keywords: Caputo derivative, Stability, Hopf Bifurcation, Dynamical Systems. 


\begin{abstract}
In this manuscript a new approach into analyzing the local stability of equilibrium points of non-linear Caputo fractional planar systems is shown. It is shown that the equilibrium points of such systems can be a stable focus or unstable focus. In addition, it is proposed that previous results regarding the stability of equilibrium points have been incorrect, the results here attempt to correct such results. Lastly, it is proposed that a Caputo fractional planar system cannot undergo a Hopf bifurcation, contrary to previous results prior. Though, it is shown that such systems can undergo a Hopf bifurcation (topologically).
\end{abstract}

\title{
1 Introduction
}

Fractional Differential Equations (FDE) have been growing in popularity in the field of applied mathematics, in particular in the field of mathematical modeling, see $[2,5,6,9-12,17]$. The primary modeling approach in the references is via Dynamical Systems with $\alpha \in(0,1)$, where $\alpha$ is the order of the derivative. Such models are particularly popular in modeling disease spread of Predator-Prey interactions (ecosystems), see $[3,7,20,21]$. Traditionally, the authors in those papers are interested in determining the qualitative behavior of the system near its equilibria points, by employing the classical theory of local stability analysis or bifurcation theory. Similarly, for the fractional case, authors attempt to do the same.

However, due to the complexity of the fractional derivative, the results obtained are not always as strong as the classical. non-fractional, case. That is to say, for the classical case the local stability theory is well developed and it is easy to justify the qualitative behavior of a system near its equilibria point, as well as provide a complete characterization of the solutions. However, for the fractional case this is not the case.

In [22] Corollary 2, it was shown that a Caputo autonomous fractional order system, with order $\alpha \in(0,1)$, cannot have a non-constant smooth periodic solution. This result is fundamental to this manuscript. In fact, we will use this result to conclude Theorem $3.2(i)$, for the case when $\alpha=\alpha^{*}:=\frac{2}{\pi}\left|\arg \left(\lambda_{1,2}\right)\right|$. As a consequence of this, we have that a Caputo autonomous fractional order system with order $\alpha \in(0,1)$ cannot undergo a Hopf bifurcation as $\alpha$ crosses the critical value $\alpha^{*}$. 
Resulting in Lemma 3.3 in [17] being incorrect. It is proposed in this manuscript, in Theorem $3.2(i)$, that, if the linearized part of (3.1) at the equilibrium point $\left(x^{*}, y^{*}\right)$ has complex eigenvalues with a positive real part, and if $\alpha=\alpha^{*}$, then the equilibrium point is to undergo a Hopf bifurcation (topologically).

Additionally, in Theorem 3.2 and its proof we show that the results obtained by the authors in [19] Theorem $4(a)$, and $(f)$ are incorrect, see remark 3.1.

\section{In summary:}

1. It is shown that the local stability of hyperbolic equilibrium points of a Caputo fractional planar system can be analyzed by studying the asymptotic behavior of the Mittag-Leffler function defined in (2.3).

2. It shown that, depending on $\alpha \in(0,1)$, that the hyperbolic equilibrium point can be an unstable focus, stable focus or locally asymptotically stable, provided that the eigenvalues are complex with a positive real part. The results for unstable focus and stable focus are new.

3. It is concluded that a Caputo autonomous fractional order system of order $\alpha \in(0,1)$ cannot undergo a Hopf bifurcation, rather that under suitable conditions, if $\alpha=\alpha^{*}$ then the hyperbolic equilibrium point undergoes a Hopf bifurcation (topologically).

\section{Preliminaries}

Definition 2.1. Let $0<\alpha<1$. The operator $J_{a}^{\alpha}$, defined on $L^{1}[a, b]$ by

$$
J_{a}^{\alpha} f(t)=\frac{1}{\Gamma(\alpha)} \int_{a}^{t}(t-x)^{\alpha-1} f(x) d x
$$

for $a \leq t \leq b$, is called the Riemann-Liouville fractional integral operator of order $\alpha$. Here, and in what follows $\Gamma(\cdot)$ is the Gamma function.

Definition 2.2. Let $0<\alpha<1$. Then, we define the Caputo fractional differential operator $c D_{a}^{\alpha}$ as

$$
c D_{a}^{\alpha} f(t):=J_{a}^{1-\alpha} f^{\prime}(t)
$$

whenever $f, f^{\prime} \in L[a, b]$. 
Definition 2.3. Let $\alpha>0, \beta>0$. The function $E_{\alpha}$, defined by

$$
E_{\alpha, \beta}(z)=\sum_{j=0}^{\infty} \frac{z^{j}}{\Gamma(\alpha j+\beta)}
$$

whenever the series converges is called the two parameter MittagLeffler function with parameters $\alpha$ and $\beta$.

Lemma 2.1. If $0<\alpha<1, \beta \in \mathbb{C}$ and $\mu \in \mathbb{R}$ such that

$$
\alpha \frac{\pi}{2}<\mu<\alpha \pi,
$$

then for a arbitrary integer $p \geq 1$ the following expansion holds:

$E_{\alpha}(z)=\frac{1}{\alpha} z^{(1-\beta) / \alpha} e^{z^{1 / \alpha}}-\sum_{k=1}^{p} \frac{z^{-k}}{\Gamma(\beta-\alpha k)}+O\left(|z|^{-1-p}\right) \quad|z| \rightarrow \infty,|\arg (z)| \leq \mu$

or

$E_{\alpha}(z)=-\sum_{k=1}^{p} \frac{z^{-k}}{\Gamma(\beta-\alpha k)}+O\left(|z|^{-1-p}\right) \quad|z| \rightarrow \infty . \mu \leq|\arg (z)| \leq \pi$

Remark 2.1. Note, that the terms

$$
\sum_{k=1}^{p} \frac{z^{-k}}{\Gamma(\beta-\alpha k)}+O\left(|z|^{-1-p}\right)
$$

become arbitrary small as $|z| \rightarrow \infty$. Fix $\beta=1$, then

$$
E_{\alpha}(z)=\frac{1}{\alpha} e^{z^{1 / \alpha}} \quad|z| \rightarrow \infty,|\arg (z)| \leq \mu
$$

\section{Local Stability Theory of Planar Frac- tional System}

In this section we provide the stability theory that we will use in the paper for Planar Fractional Systems. Specifically, we build on the the 
classical theory and extend the results to the fractional case. We only consider the case when $\alpha \in(0,1)$.

$$
\left\{\begin{array}{l}
c D_{0}^{\alpha} x(t)=f(x, y), \\
c D_{0}^{\alpha} y(t)=g(x, y),
\end{array}\right.
$$

subject to the initial condition:

$$
(x(0), y(0))=\left(x_{0}, y_{0}\right)
$$

where $f, g \in C^{1}\left(\mathbb{R}^{2}\right)$.

Since, $f, g \in C^{1}\left(\mathbb{R}^{2}\right)$, it is well known that for any $\left(x_{0}, y_{0}\right) \in \mathbb{R}^{2}$ the initial value problem (3.1) has a unique solution, see [4].

We denote by $A(x, y)$ the Jacobian matrix of $f$ and $g$ at $(x, y)$, that is,

$$
A(x, y)=\left(\begin{array}{ll}
\frac{\partial f}{\partial x} & \frac{\partial f}{\partial y} \\
\frac{\partial g}{\partial x} & \frac{\partial g}{\partial y}
\end{array}\right),
$$

and by $|A(x, y)|$ and $\operatorname{tr}(A(x, y))$ the determinant and trace of $A(x, y)$, respectively.

Below we provide the definitions for a cyccle or periodic orbit, and Hopf bifurcation.

Definition 3.1. A point $\left(x^{*}, y^{*}\right) \in \mathbb{R}^{2}$ is called an equilibrium point of (3.1) if $f\left(x^{*}, y^{*}\right)=0$ and $g\left(x^{*}, y^{*}\right)=0$.

Below we define the linearized system of (3.1) about the equilibrium point $\left(x^{*}, y^{*}\right)$.

Definition 3.2. Let $A$ be the matrix defined in (3.2) is evaluated at the equilibrium point $\left(x^{*}, y^{*}\right)$. Then,

$$
c D_{0}^{\alpha} X=A X,
$$

where $X=(x, y)^{T}$, is the linearization of system (3.1) at the equilibrium point $\left(x^{*}, y^{*}\right)$.

Definition 3.3. A cycle or periodic orbit of (3.1) is any closed trajectory curve of (3.1) which is not an equilibrium point of (3.1). A limit cycle $\Gamma$ of a planar system is a cycle of (3.1) which belongs to the $\alpha$ or $\omega$ limit set of some trajectory of (3.1) other than $\Gamma$. If a cycle $\Gamma$ is the $\omega$ limit set of every trajectory in some neighborhood of $\Gamma$, then $\Gamma$ is a stable limit cycle. 
Definition 3.4. Hopf bifurcation is a local bifurcation in which a steady state of a dynamical system changes its stability, so that the appearance or disappearance of a periodic orbit occurs.

The following Lemma can be found in [22] as Corollary 2.

Lemma 3.1. The Caputo fractional order system defined in (3.1), where $\alpha \in(0,1)$, cannot have any non-constant smooth periodic solutions.

As a direct consequence of Lemma 3.1 above, we obtain the following result.

Proposition 3.1. (1) The Caputo fractional order system defined in (3.1), where $\alpha \in(0,1)$, cannot have a limit cycle.

(2) The Caputo fractional order system defined in (3.1), where $\alpha \in(0,1)$, cannot undergo a Hopf bifurcation.

Proof. (1) Suppose for a contradiction that (3.1) does have a limit cycle. Then, there exists a cycle, $\Phi$, of (3.1) such that the cycle belongs to the $\alpha$ or $\omega$ limit set. Furthermore, the cycle cannot be constant solution of (3.1), since it is not an equilibrium point of (3.1). Thus, the cycle or periodic orbit through $(x, y)$ is non-constant, and for each $t \in[0, \infty)$, there exists a $\tau \in(0, \infty)$ such that

$$
\Phi(t, x, y)=\Phi(t+\tau, x, y)
$$

However, by Lemma 3.1, we have that $\Phi$ cannot satisfy (3.1). Thus, the cycle $\Phi$ cannot be a cycle of (3.1). The result follows.

The result (2) follows immediatly from definition 3.4, and from the result above.

The following Lemma is a special case $(n=2)$ of Lemma 3.2 in [17].

Lemma 3.2. [17] Let $\left(x^{*}, y^{*}\right)$ be an equilibrium point of (3.1) and $A$ be defined as in (3.2). Let $\lambda_{1}$ and $\lambda_{2}$ be the eigenvalues of $A$. Then, the following assertions hold.

(1) The equilibrium point $\left(x^{*}, y^{*}\right)$ is locally asymptotically stable if and only if $\left|\arg \left(\lambda_{1,2}\right)\right|>\frac{\alpha \pi}{2}$.

(2) The equilibrium point $\left(x^{*}, y^{*}\right)$ is stable if and only if $\left|\arg \left(\lambda_{1,2}\right)\right| \geq$ $\frac{\alpha \pi}{2}$ and the eigenvalues with $\left|\arg \left(\lambda_{1,2}\right)\right|=\frac{\alpha \pi}{2}$ have the same geometric multiplicity and algebraic multiplicity. $\frac{\alpha \pi}{2}$.

(3) The equilibrium point $\left(x^{*}, y^{*}\right)$ is unstable if and only if $\left|\arg \left(\lambda_{1,2}\right)\right|<$ 
Lemma 3.3. [16][Theorem 3] If the origin $(0,0)$ is a hyperbolic equilibrium point of (3.1), then vector field $(f(x, y), g(x, y))$ is topologically equivalent with its linearization vector field given by the linear system $c D_{0}^{\alpha} X=A X$ in the neighborhood of the origin $(0,0)$.

The following Theorem follows from Lemma 3.2, where the conditions are expressed in terms of $\operatorname{tr}\left(A\left(x^{*}, y^{*}\right)\right)$, and $\left|A\left(x^{*}, y^{*}\right)\right|$.

Theorem 3.1. If $\left(x^{*}, y^{*}\right)$ is a equilibrium point of (3.1), then the following assertions hold.

(i) If $\left|A\left(x^{*}, y^{*}\right)\right|<0$, then $\left(x^{*}, y^{*}\right)$ is unstable. (3.1).

(ii) If $\left|A\left(x^{*}, y^{*}\right)\right|>0, \operatorname{tr}\left(A\left(x^{*}, y^{*}\right)\right)>0$ and $\left(\operatorname{tr}\left(A\left(x^{*}, y^{*}\right)\right)\right)^{2}-4\left|A\left(x^{*}, y^{*}\right)\right| \geq$ 0 , then $\left(x^{*}, y^{*}\right)$ is unstable.

(iii) If $\left|A\left(x^{*}, y^{*}\right)\right|>0, \operatorname{tr}\left(A\left(x^{*}, y^{*}\right)\right)<0$, then $\left(x^{*}, y^{*}\right)$ is Locally Asymptotically stable.

The Theorem below is new.

Theorem 3.2. Let $\left(x^{*}, y^{*}\right)$ be an equilibrium point of (3.1). Let $\lambda_{1}$ and $\lambda_{2}$ be eigenvalues of the matrix $A$ defined in (3.2) and suppose that $\lambda_{1}=a+i b$ and $\lambda_{2}=a-i b$, with $b \neq 0$.

(i) If $\left|A\left(x^{*}, y^{*}\right)\right|>0, \operatorname{tr}\left(A\left(x^{*}, y^{*}\right)\right)>0$ and $\left(\operatorname{tr}\left(A\left(x^{*}, y^{*}\right)\right)\right)^{2}-4\left|A\left(x^{*}, y^{*}\right)\right|<$ 0 , then $\left(x^{*}, y^{*}\right)$ is an unstable focus of (3.1) for $\alpha \in\left(\alpha^{*}, 1\right)$; stable focus of (3.1) for $\alpha \in\left(0, \alpha^{*}\right)$; a Hopf bifurcation (topologically), if $\alpha=\alpha^{*}$. Where $\alpha^{*}=(2 / \pi)\left|\arg \left(\lambda_{1}\right)\right|$.

(ii) If $\left|A\left(x^{*}, y^{*}\right)\right|>0, \operatorname{tr}\left(A\left(x^{*}, y^{*}\right)\right)<0$ and $\left(\operatorname{tr}\left(A\left(x^{*}, y^{*}\right)\right)\right)^{2}-4\left|A\left(x^{*}, y^{*}\right)\right|<$ 0 , then $\left(x^{*}, y^{*}\right)$ is a stable focus of (3.1) for $\alpha \in\left(\frac{1}{2}, 1\right)$; locally asymptotically stable for $\alpha \in\left(0, \frac{1}{2}\right]$.

Proof. By Lemma (3.3) we can study (3.3) to determine the qualitative behavior of the equilibrium point $\left(x^{*}, y^{*}\right)$. If $\left(x^{*}, y^{*}\right) \neq(0,0)$, then we can use the following substitution

$$
x_{1}=x-x^{*}, \quad y_{1}=y-y^{*},
$$

to translate the equilibrium point $\left(x^{*}, y^{*}\right)$ to the origin.

Define $\left|\arg \left(\lambda_{1,2}\right)\right|$ to be the argument of the eigenvalues $\lambda_{1}$, and $\lambda_{2}$, which are equal since the eigenvalues are complex conjugates. Additionally, since $b \neq 0$, then there exists a $\mu \in\left(\frac{\alpha \pi}{2}, \alpha \pi\right)$ such that $\left|\arg \left(\lambda_{1,2}\right)\right| \leq \mu$. Moreover, $\lambda_{1} \neq \lambda_{2}$, thus the general solution, $x$, to (3.3) can be expressed as follows 


$$
X(t)=c_{1} u_{1} E_{\alpha}\left(\lambda_{1} t^{\alpha}\right)+c_{2} u_{2} E_{\alpha}\left(\lambda_{2} t^{\alpha}\right),
$$

where $c_{1}$ and $c_{2}$ are constants and $u_{1}$ and $u_{2}$ are eigenvectors corresponding to the eigenvalues $\lambda_{1}$ and $\lambda_{2}$, respectively. Since, we are interested in the qualitative behavior solutions about the equilibrium points, instead of transient dynamics, we can consider the behavior of the solution

$$
X(t)=c_{1} u_{1} E_{\alpha}\left(\lambda_{1} t^{\alpha}\right)+c_{2} u_{2} E_{\alpha}\left(\lambda_{2} t^{\alpha}\right), \quad \text { as } t \rightarrow \infty .
$$

Since, there exists a $\mu \in\left(\frac{\alpha \pi}{2}, \alpha \pi\right)$ such that $\left|\arg \left(\lambda_{1,2}\right)\right| \leq \mu$, by Lemma (2.1)

$$
E_{\alpha}\left(\lambda_{1} t^{\alpha}\right)=(1 / \alpha) e^{\left(\lambda_{1} t^{\alpha}\right)^{1 / \alpha}} \quad \text { as } t \rightarrow \infty \text { and }\left|\arg \left(\lambda_{1,2}\right)\right| \leq \mu .
$$

Define $\alpha_{1}:=\left\lceil\frac{1}{\alpha}\right\rceil$, then $\alpha_{1} \in \mathbb{Z}_{+}$.

$$
\begin{aligned}
(1 / \alpha) e^{\left(\lambda_{1} t^{\alpha}\right)^{1 / \alpha}} & =\alpha_{1} e^{(a+i b)^{\alpha_{1}} t} \\
& =\alpha_{1} e^{\left(\sum_{j=0}^{\alpha_{1}}\left(\begin{array}{c}
n \\
j
\end{array}\right) a^{\alpha_{1}}(i b)^{\alpha_{1}-j}\right) t} \\
& =\alpha_{1} e^{\left(\left(\begin{array}{c}
\alpha_{1} \\
0
\end{array}\right) a^{\alpha_{1}}+\left(\begin{array}{c}
\alpha_{1} \\
1
\end{array}\right) a^{\alpha_{1}-1}(i b)+\ldots+(i b)^{\alpha_{1}}\right) t} .
\end{aligned}
$$

Note that, in principle, the terms

$$
\left(\begin{array}{c}
\alpha_{1} \\
0
\end{array}\right) a^{\alpha_{1}}+\left(\begin{array}{c}
\alpha_{1} \\
1
\end{array}\right) a^{\alpha-1}(i b)+\ldots+(i b)^{\alpha_{1}}
$$

can be split up into real valued terms and complex valued terms. Thus,

$$
\left(\begin{array}{c}
\alpha_{1} \\
0
\end{array}\right) a^{\alpha_{1}}+\left(\begin{array}{c}
\alpha_{1} \\
1
\end{array}\right) a^{\alpha-1}(i b)+\ldots+(i b)^{\alpha_{1}}=\omega+i \beta \quad \text { for some } \omega, \beta \in \mathbb{R}
$$

Hence, 


$$
\begin{aligned}
& =\alpha_{1} e^{\left(\left(\begin{array}{c}
\alpha_{1} \\
0
\end{array}\right) a^{\alpha_{1}}+\left(\begin{array}{c}
\alpha_{1} \\
1
\end{array}\right) a^{\alpha_{1}-1}(i b)+\ldots+(i b)^{\alpha_{1}}\right) t} \\
& =\alpha_{1} e^{(\omega+i \beta) t}=\alpha_{1} e^{\omega t}(\cos (\beta t)+i \sin (\beta t)) .
\end{aligned}
$$

Note that, the qualitative behaviour of (3.5) is the same the qualitative behaviour of (3.7), which depend only on the eigenvalues and $\alpha$. See Lemma 3.2.

Similarly,

$$
(1 / \alpha) e^{\left(\lambda_{2} t^{\alpha}\right)^{1 / \alpha}}=\alpha_{1} e^{\omega t}(\cos (\beta t)-i \sin (\beta t))
$$

Thus, (3.4) can be expressed as

$$
\begin{aligned}
X(t) & =c_{1} u_{1}\left[\alpha_{1} e^{\omega t}(\cos (\beta t)+i \sin (\text { betat }))\right] \\
& +c_{2} u_{2}\left[\alpha_{1} e^{\omega t}(\cos (\beta t)-i \sin (\beta t))\right]
\end{aligned}
$$

as $t \rightarrow \infty$ and $\left|\arg \left(\lambda_{1,2}\right)\right| \leq \mu$.

Thus, from (3.8) we can see that the solutions, can be expressed strictly as real valued solutions, similarly as the classical case. In addition, the solutions behave in an oscillatory manner, similarly as the classical case, qualitatively.

Next, recall that the eigenvalues for a planar system can can be represented as

$$
\begin{aligned}
& \lambda_{1}=\frac{\operatorname{tr}(A(x, y))+\sqrt{\operatorname{tr}(A(x, y))^{2}-4 \operatorname{det}(A(x, y))}}{2}, \\
& \lambda_{2}=\frac{\operatorname{tr}(A(x, y))-\sqrt{\operatorname{tr}(A(x, y))^{2}-4 \operatorname{det}(A(x, y))}}{2} .
\end{aligned}
$$

(1) If $\operatorname{tr}(A(x, y))>0$, and $\operatorname{tr}(A(x, y))^{2}-4 \operatorname{det}(A(x, y))<0$, then the eigenvalues are complex conjugates. Note that the eigenvalues are independent of $\alpha$, so $\left|\arg \left(\lambda_{1,2}\right)\right|$ is fixed. Specifically, the term $\alpha^{*}=\frac{2}{\pi}\left|\arg \left(\lambda_{1,2}\right)\right|$ is fixed. 
(i) Suppose that $\alpha \in\left(\alpha^{*}, 1\right)$, then $\left|\arg \left(\lambda_{1,2}\right)\right|<\frac{\alpha \pi}{2} \leq \mu<\alpha \pi$. From (3.8) the solution has a oscillatory behavior. This together with Lemma (3.2) allows us to conclude that $\left(x^{*}, y^{*}\right)$ is an unstable focus.

(ii) Suppose that $\alpha \in\left(0, \alpha^{*}\right)$, then there exists a $\mu \in\left(\frac{\alpha \pi}{2}, \alpha \pi\right)$ such that $\frac{\alpha \pi}{2}<\left|\arg \left(\lambda_{1,2}\right)\right| \leq \mu<\alpha \pi$. From (3.8) the solution has a oscillatory behavior. This together with Lemma (3.2) allows us to conclude that $\left(x^{*}, y^{*}\right)$ is a stable focus.

(iii) Suppose that $\alpha=\alpha^{*}$, then there exists a $\mu \in\left(\frac{\alpha \pi}{2}, \alpha \pi\right)$ such that $\left|\arg \left(\lambda_{1,2}\right)\right|=\frac{\alpha \pi}{2}<\mu$. From (3.8) the solution has a oscillatory behavior. Furthermore, by Lemma (3.2) we have that the solutions are stable. Hence, the equilibrium point $\left(x^{*}, y^{*}\right)$ cannot be a stable focus. By Lemma 3.1, and Proposition 3.1, we have that (3.1) cannot have a limit cycle, and hence the equilibrium point $\left(x^{*}, y^{*}\right)$ cannot undergo a Hopf bifurcation. However, by (3.8), the solutions have an oscillatory behaviour. Thus, from above, and by logical necessity, the equilibrium point $\left(x^{*}, y^{*}\right)$ undergoes a Hopf bifurcation (topologically). That is to say, when $\alpha=\alpha^{*}$, the solutions of (3.1) behave, topologically, as a limit cycle. Specifically, a stable limit cycle, since by Lemma 3.2 the equilibrium point $\left(x^{*}, y^{*}\right)$ is stable under the conditions in $(i i)$ for $\alpha=\alpha^{*}$.

(2) If $\operatorname{tr}(A(x, y))<0$, and $\operatorname{tr}(A(x, y))^{2}-4 \operatorname{det}(A(x, y))<0$, then the eigenvalues are complex conjugates, and $\left|\arg \left(\lambda_{1,2}\right)\right|>\frac{\pi}{2}$. Let $\alpha \in\left(\frac{1}{2}, 1\right)$ and $\mu \in\left(\frac{\alpha \pi}{2}, \alpha \pi\right)$. Then, there exists a $\mu \in\left(\frac{\alpha \pi}{2}, \alpha \pi\right)$ such that $\frac{\alpha \pi}{2}<\frac{\pi}{2}<\left|\arg \left(\lambda_{1,2}\right)\right|<\mu<\alpha \pi$. From (3.8) the solution has a oscillatory behavior. This together with Lemma (3.2) allows us to conclude that $\left(x^{*}, y^{*}\right)$ is a stable focus.

Remark 3.1. In Theorem 3.2 we provide a new method for determining the qualitative behavior of solutions near equilibrium point $\left(x^{*}, y^{*}\right)$. In particular, we use the asymptotic expansion properties of the Mittag-Leffler functions to achieve the results. Furthermore, we show that under suitable conditions the equilibrium point $\left(x^{*}, y^{*}\right)$ undergoes a Hopf bifurcation (topologically). This result is new and has not been presented elsewhere.

In fact, the condition $\alpha=\alpha^{*}$, has been misrepresented in the literature, see $[17,19,23]$. The authors in $[17,23]$ claimed that equilibrium point $\left(x^{*}, y^{*}\right)$ of (3.1) is a Hopf bifurcation if $\alpha=\alpha^{*}$. However, this is not correct, if the claim is to be true, then it is to follow that (3.1) undergoes a limit cycle, which from Proposition 3.1 (1) cannot be the 
case. As we have shown the solutions only "mimic" the behaviour of a limit cycle. Thus, it is not possible to treat $\alpha$ as bifurcation parameter in this case. Nor, is it correct to claim that this leads to (3.1) undergoing a Hopf bifurcation. The authors in [19] claimed that the equilibrium point $\left(x^{*}, y^{*}\right)$ is a stable node, if $\alpha=\alpha^{*}$. This claim is also not correct, in fact from the proof above, we see that when $\alpha=\alpha^{*}$ the solutions have a oscillatory behaviour. Additionally, Theorem (4) $(f)$ in [19] is not correct. In fact, the author states that if all the eigenvalues are complex and satisfy $\left|\arg \left(\lambda_{1,2}\right)\right|>\frac{\alpha \pi}{2}$, then the equilibrium point $\left(x^{*}, y^{*}\right)$ is a stable focus of $(3.1)$. However, this can only be concluded in its entirety for the linear system (3.3). It does not follow that it holds completely for the system (3.1). Indeed, consider the case when the complex eigenvalues have a zero real part, then $\left|\arg \left(\lambda_{1,2}\right)\right|=\frac{\pi}{2} \geq \frac{\alpha \pi}{2}$. However, since the eigenvalues zero real parts, then this equilibrium point is a non hyperbolic equilibrium point, and the linearization Lemma 3.3 does not apply.

Lastly, due to the conditions associated with the asymptotic expansion of the Mittag-Leffler function, we cannot conclude that under (ii) the equilibrium point $\left(x^{*}, y^{*}\right)$ is a stable focus for $\alpha \in\left(0, \frac{1}{2}\right]$.

Below we apply Theorem $3.2(i)$ to demonstrate the result.

Example 3.1. Consider the following Caputo-fractional Lienard system.

$$
\left\{\begin{array}{l}
c D_{0}^{\alpha} x(t)=y-\frac{x^{3}-x}{x^{2}+1} \\
c D_{0}^{\alpha} y(t)=-x
\end{array}\right.
$$

Then, $(0,0)$ is an equilibrium point, and the eigenvalues of the linearized system near the origin is given by

$$
\lambda_{1}=\frac{1}{2}-\frac{i \sqrt{3}}{2} \text {, and } \lambda_{2}=\frac{1}{2}+\frac{i \sqrt{3}}{2},
$$

and $\alpha^{*}=2 / 3$. Using fde12 solver, see [18], we obtain the following numerical simulation 


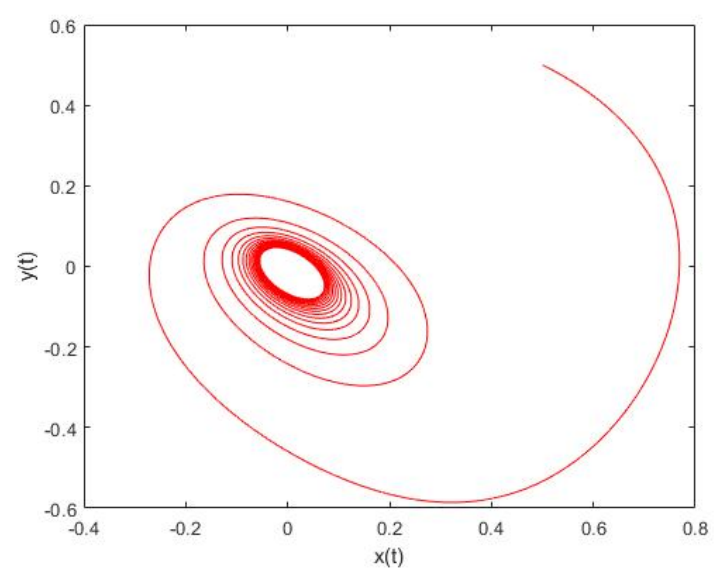

Conclusion: In this manuscript a new method for analyzing the local stability of hyperbolic equilibrium points for a Caputo fractional planar system was presented, see the proof of Theorem 3.2. It was shown that the equilibrium point could be an unstable focus, stable focus or locally asymptotically stable, under suitable conditions. Furthermore, it was shown that a hyperbolic equilibrium point of (3.1) undergoes a Hopf bifrucation (topologically). The future work in this area would be to determine the stability of non-hyperbolic equilibria points of (3.1). 


\section{References}

[1] Diethelm, K. (2010). The Analysis of fractional differential equations. Berlin: Springer.

[2] Z. M Odibat. Analytical study on linear systems of fractional differential equations. Computers and Mathematics with Applications 59(2010) 1171-1183.

[3] C. Chow, M. Hoti, C.L Li, and K.Q Lan. Local stability analysis on Lotka-Volterra predator-prey mmodels with prey refuge and harvesting. Math. Methods Appl Sci (2018) 1-22.

[4] Podlubny, I. (1999). Fractional Differential Equations. Mathematics in Science and Engineering, vol 198.

[5] D. Qian, C. Li, R.P Agarwal, P.J.Y Wong. Stability analysis of fractional differential system with Riemann-Liouville derivative. Mathematical and Computer Modelling (2010)862-874.

[6] D. Matignon. Stability results for fractinal differential equations with applications to control processing. Computational Engineering in Systems and Application Multiconference, IMACS, IEEESMC, Lille, France, Vol 2, 1996, pp. 963-968.

[7] L.Veen, M. Hoti. Automatic Detection of Saddle-NodeTranscritical Interactions. International Journal of Bifurcation and Chaos. Vol 29, No. 8 (2019) 1950104.

[8] M. Kirane, B. T. Torebek. A Lyapunov type inequality for a fractional boundary value problem with Caputo-Fabrizio Derivative. Journal of Mathematical Inequalities. (2018), 1005-1012.

[9] Kumar. D, Singh. J, Qurashi. M and Baleanu. D. Analysis of logistic equation pertaining to a new fractional derivative with a non-singular kernel. Advances in Mechanical Engineering. Vol $9(2)(2017), 1-8$.

[10] Boukhouima. A, Hattaf. K and Yousif. N. Dynamics of a Fractional Order HIV Infection Model with Specific Functional Response and Cure Rate. International Journal of Differential Equations, (2017), 1-8.

[11] Rostamy. D and Mottaghi. E. Stability analysis of a fractional order epidemics model with multiple equilibriums. Advances in Difference Equations. 2016:170. 
[12] El-Saka. H.A.A. The fractional order SIS-epidemic model with a variable population size. Journal of Egyptian Mathematical Society. 2014 (22), 50-54.

[13] J. R. L. Webb, Weakly singular Gronwall inequalities and applications to fractional differential equations, J. Math. Anal. Appl 471 (2019), 692-711.

[14] K. Q. Lan and W. Lin, Positive solutions of systems of Caputo fractional differential equations, Communications in Applied Analysis 17(2013) (1), 61-86.

[15] J. R. L. Webb, Initial value problems for Caputo fractional equations with singular nonlinearities, Electronic Journal of Differential Equations 117 (2019), 1-32.

[16] C. Li and Y. Ma. Fractional Dynamical Systems and Its Linearization Theorem, Nonlinear Dynamics (2013).

[17] B. Tang. Dynamics for a fractional predator prey model with group defense, Nature-Scientific Reports (2020).

[18] R. Garrappa. Numerical Solutions of Fractional Differential Equations: A Survey and a Software Tutorial, Mathematics (2018).

[19] F. Keshtkar, G. Erjaeem, and M. Boutefnouchet. On Stability of Equilibrium points in Nonlinear Fractional Differential Equations and Fractional Hamiltonian Systems Complexity (2014).

[20] B.W Kooi, D. Bontje, G.A.K. Van Voorn, S.A.L.M. Kooijman. Sublethal toxic effects in a simple aquatic food chain. Ecological Modelling 212 (2008), 304-318.

[21] Li. Sheng, N. S Joseph, S.H Ian,J.S Koopman. Dynamics and Control of Infections Transmitted From Person to Persons Through the Environment American Journal of Epidemiology (2009).

[22] Tavazoei, M.S. A note on fractional-order derivatives of periodic functions. Automatica 46, 945-948 (2010).

[23] Li, X. and Wu, R. C. Hopf bifurcation analysis of a new commensurate fractional-order hyperchaotic system. Nonlinear Dyn 78, 279-288 (2014). 